PROCEEDINGS OF THE

AMERICAN MATHEMATICAL SOCIETY

Volume 127, Number 7, Pages 2183-2192

S 0002-9939(99)04731-0

Article electronically published on February 17, 1999

\title{
PSEUDO-ANOSOV HOMEOMORPHISMS WITH QUADRATIC EXPANSION
}

\author{
J. FRANKS AND E. RYKKEN \\ (Communicated by Mary Rees)
}

\begin{abstract}
We show that if $f: M \rightarrow M$ is a pseudo-Anosov homeomorphism on an orientable surface with oriented unstable manifolds and a quadratic expanding factor, then there is a hyperbolic toral automorphism on $\mathbb{T}^{2}$ and a map $h: M \rightarrow \mathbb{T}^{2}$ such that $h$ is a semi-conjugacy and $(M, h)$ is a branched covering space of $\mathbb{T}^{2}$. We also give another characterization of pseudo-Anosov homeomorphisms with quadratic expansion in terms of the kinds of Euclidean foliations they admit which are compatible with the affine structure associated to $f$.
\end{abstract}

\section{INTRODUCTION AND DEFINITIONS}

In this article we consider pseudo-Anosov homeomorphisms on an orientable surface with oriented stable and unstable manifolds. In the first section we recall the basic definitions and background theorems. The second section is devoted to the proof of the following theorem.

Theorem 2.3. If $f: M \rightarrow M$ is a pseudo-Anosov homeomorphism on an orientable surface with oriented unstable manifolds and a quadratic expanding factor, then there is a hyperbolic toral automorphism $B$ on $\mathbb{T}^{2}$ and a map $h: M \rightarrow \mathbb{T}^{2}$ such that $h$ is a semi-conjugacy from $f$ to $B$ and $(M, h)$ is a branched covering space of $\mathbb{T}^{2}$. Moreover the map $h$ restricted to the complement of the singularities of $f$ is a locally affine map from the flat Euclidean structure on $M$ to the natural affine structure on $\mathbb{T}^{2}$.

A final section is devoted to another characterization of pseudo-Anosov homeomorphisms with quadratic expansion. In particular, we define the notion of a rational foliation associated to $f$ to be an affine foliation compatible with the Euclidean structure associated with $f$ with the property that all its leaves are compact and the property that the non-singular leaves form a finite union of annuli whose widths are rationally related. A more formal definition is given in Definition 3.2. We then prove the following.

Theorem 3.3. Suppose $f: M \rightarrow M$ is a pseudo-Anosov homeomorphism on an orientable surface with oriented stable and unstable manifolds, then the following are equivalent:

1) The expansion factor $\lambda$ of $f$ is quadratic.

Received by the editors August 22, 1997 and, in revised form, October 1, 1997.

1991 Mathematics Subject Classification. Primary 58F15.

(C)1999 American Mathematical Society 
2) There exists an $m \in \mathbb{R}$ such that the slope foliation $\mathcal{F}_{m}$ of $M$ is rational.

3) There exists a dense set of $m \in \mathbb{R}$ such that the slope foliation $\mathcal{F}_{m}$ of $M$ is rational.

Definition 1.1. Let $M$ be a compact two-dimensional orientable manifold with a flat Euclidean structure with a finite set $S$ of isolated cone singularities [7]. A pseudo-Anosov homeomorphism on $\mathrm{M}$ is a homeomorphism $f: M \rightarrow M$ such that there exist two perpendicular foliations $W^{u}(f)$ and $W^{s}(f)$ and a real number $\lambda>1$ with the property that the image under $f$ of a leaf is a leaf and:

1) $d(f(x), f(y))=\lambda d(x, y)$ if $x, y$ are in the same leaf of $W^{u}(f)$,

2) $d(f(x), f(y))=\frac{1}{\lambda} d(x, y)$ if $x, y$ are in the same leaf of $W^{s}(f)$.

We call $W^{u}(f)$ the unstable foliation, $W^{s}(f)$ the stable foliation, and $\lambda$ the expanding factor of $f$.

A pseudo-Anosov homeomorphism $f$ is hyperbolic and locally affine. That is, in local coordinates, which are compatible with the flat Euclidean structure, the map $f$ is affine except at points of $S$. Furthermore, $f(S)=S$ and a singularity of angle $k \pi$ maps to a singularity of angle $k \pi$.

Definition 1.2. A rectangle $R$ in $M$ is the image of a closed Euclidean rectangle $\widetilde{R}$ under a continuous map $\pi$ such that $\pi$ is a one-to-one Euclidean map on the interior of $\widetilde{R}$.

Definition 1.3. Let $W^{u}(x, f)$ be the leaf of the unstable foliation which contains $x$. Let $W^{s}(x, f)$ be the leaf of the stable foliation which contains $x$. For $x \in \operatorname{int} R$, let $W^{u}(x, f, R)$ be the component of $W^{u}(x, f) \cap R$ which contains $x$ and define $W^{s}(x, f, R)$ similarly. Let the width of a rectangle $R_{i}$ be given by $r_{i}=$ length of $W^{s}\left(x, f, R_{i}\right)$, where $x \in R_{i}$. Let the length of a rectangle $R_{i}$ be given by $l_{i}=$ length of $W^{u}\left(x, f, R_{i}\right)$, where $x \in R_{i}$. Let $W_{\epsilon}^{u}(x, f)$ be the component of $W^{u}(x, f) \cap B(x, \epsilon)$ which contains $x$, where $B(x, \epsilon)$ denotes the ball of radius $\epsilon$ centered at $x$. Define $W_{\epsilon}^{s}(x, f)$ similarly.

Definition 1.4. Suppose $f$ is a pseudo-Anosov homeomorphism on $M$. A Markov partition for $f$ on $M$ is a finite covering of $M$ by rectangles, $\left\{R_{1}, R_{2}, \ldots, R_{n}\right\}$, such that:

1) For $i \neq j$, int $R_{i} \cap i n t R_{j}=\emptyset$.

2) If $x \in \operatorname{int} R_{i}$ and $f(x) \in \operatorname{int} R_{j}$, then $f\left(W^{u}\left(x, f, R_{i}\right)\right) \supset W^{u}\left(f(x), f, R_{j}\right)$

and $f\left(W^{s}\left(x, f, R_{i}\right)\right) \subset W^{s}\left(f(x), f, R_{j}\right)$.

Definition 1.5. We define the Markov matrix for a Markov partition $\mathcal{P}$ with $n$ rectangles to be the $n \times n$ matrix given by

$$
M_{i j}=\text { the number of components of intf }\left(W^{u}\left(x_{j}, f, R_{j}\right)\right) \cap i n t W^{s}\left(x_{i}, f, R_{i}\right)
$$

where $x_{i} \in \operatorname{int} R_{i}$ and $x_{j} \in$ int $R_{j}$ for $1 \leq i, j \leq n$.

The matrix does not depend on the choice of $x_{i}$ or $x_{j}$ and $M_{i, j}$ should be thought of as the number of times $\operatorname{int} f\left(R_{j}\right)$ crosses $\operatorname{int} R_{i}$.

Let $\mathcal{M}$ be the Markov matrix for a Markov partition of a pseudo-Anosov homeomorphism. Since $\mathcal{M} \geq 0$ is mixing, by the Perron-Frobenius theorem [5], we have that $\mathcal{M}$ has a unique, real and positive largest eigenvalue which exceeds the moduli of all the other eigenvalues. To this maximal eigenvalue there corresponds an eigenvector with positive coordinates. Moreover, any irreducible, non-negative matrix cannot have two linearly independent non-negative eigenvectors. Thus if we can 
find an eigenvalue of $\mathcal{M}$ with positive eigenvector, then it must be the unique, real and positive largest eigenvalue of $\mathcal{M}$. The following result is well known and an easy computation.

Theorem 1.6. Let $f$ be a pseudo-Anosov homeomorphism with expanding factor $\lambda$ and let $\mathcal{P}$ be a Markov partition for $f$ with $n$ rectangles with Markov matrix $\mathcal{M}$. If we let $r=\left\{r_{1}, r_{2}, \ldots, r_{n}\right\}$ where $r_{i}$ is the width of the rectangle $R_{i}$ and let $l=$ $\left\{l_{1}, l_{2}, \ldots, l_{n}\right\}$ where $l_{i}$ is the length of the rectangle $R_{i}$, then $r$ is a right eigenvector for $\mathcal{M}$ with eigenvalue $\lambda$, and $l$ is a left eigenvector for $\mathcal{M}$ with eigenvalue $\lambda$. Hence $\lambda$ is the unique, real and positive largest eigenvalue of $\mathcal{M}$.

The next result, known in stochastic processes, is a general result about nonnegative square matrices with integer entries that we will apply to Markov matrices.

Proposition 1.7. Suppose $\mathcal{A}$ is an $n \times n$ matrix with non-negative integer entries and suppose $\mathcal{A}$ has a real and positive, largest eigenvalue $\lambda$ of multiplicity 1 which exceeds the moduli of all other eigenvalues. Then

$$
\lim _{n \rightarrow \infty} \frac{\mathcal{A}^{n}}{\lambda^{n}}=\mathcal{M}=r l
$$

where $\mathcal{M}$ is a constant matrix and $r$ and $l$ are appropriately scaled right and left eigenvectors for $\lambda$, respectively. This will imply that

$$
\lim _{n \rightarrow \infty} \frac{\mathcal{A}_{i i}^{n}}{\sum_{j} \mathcal{A}_{j j}^{n}}=\frac{r_{i} l_{i}}{\sum_{j} r_{j} l_{j}} .
$$

For a proof see pp. 546-547 of [6].

\section{MAIN THEOREM}

If $f: M \rightarrow M$ is a pseudo-Anosov homeomorphism on an orientable surface with oriented unstable manifolds and a quadratic expanding factor, then there is a basis for $H_{1}(M)$ such that $f_{* 1}: H_{1}(M) \rightarrow H_{1}(M)$ is given by the matrix

$$
\mathcal{A}=\left[\begin{array}{ll}
C & X \\
0 & B
\end{array}\right]
$$

where $\lambda$ is an eigenvalue of $B$, a $2 \times 2$ integer matrix. Thus there is a map $\phi$ : $\pi_{1}(M) \rightarrow \pi_{1}\left(\mathbb{T}^{2}\right)=H_{1}\left(\mathbb{T}^{2}\right)$ given by Abelianization and then projection onto the last two coordinates which makes the following diagram commute:

$$
\begin{array}{llc}
\pi_{1}(M) & \stackrel{\phi}{\longrightarrow} & \pi_{1}\left(\mathbb{T}^{2}\right)=H_{1}\left(\mathbb{T}^{2}\right) \\
\downarrow f_{\#} & & \downarrow B_{\#} \\
\pi_{1}(M) & \stackrel{\phi}{\longrightarrow} & \pi_{1}\left(\mathbb{T}^{2}\right)=H_{1}\left(\mathbb{T}^{2}\right)
\end{array}
$$

Since $B: \mathbb{T}^{2} \rightarrow \mathbb{T}^{2}$ is a hyperbolic toral automorphism, it is a $\pi_{1}$ diffeomorphism. Hence there exists $h: M \rightarrow \mathbb{T}^{2}$ such that $B \circ h=h \circ f$ with $h_{\#}=\phi$. [3] Since $M$ is compact, $h$ will be uniformly continuous.

Lemma 2.1. $h$ is onto.

Proof. Notice that unstable manifolds map to unstable manifolds under $h$, and consider the image under $h$ of the unstable manifold of a base point in $M$. Since the unstable manifold is dense, in order for $h$ to be non-trivial, the image must contain at least two points and hence the interval between them. Therefore the image contains an interval in the unstable manifold of a point on the torus. Thus 


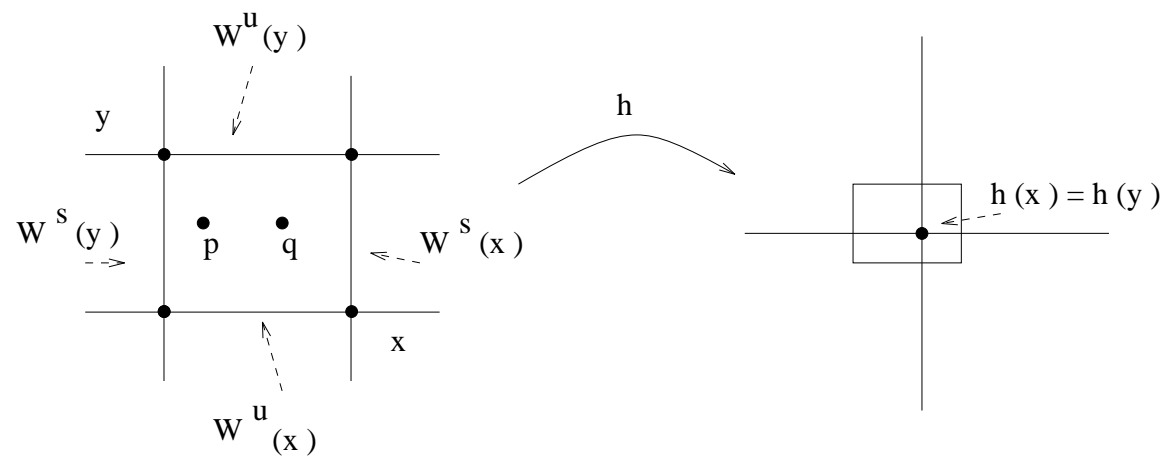

FigURE 1

the image of $M$ is a compact, invariant set under $B$ which contains a $C^{2}$ arc, and hence the image contains a torus of dimension greater than or equal to two [4]. Thus the image of $M$ under $h$ is the whole torus.

Lemma 2.2. There exists a $\delta>0$ such that given any rectangle of diameter less than $\delta, h$ restricted to that rectangle is a homeomorphism onto its image.

Proof. Choose $\epsilon$ sufficiently small that the intersections $W_{\epsilon}^{u}(y, f) \cap W_{\epsilon}^{s}(x, f)$ and $W_{\epsilon}^{u}(w, B) \cap W_{\epsilon}^{s}(v, B)$ consist of at most one point for any $x, y$ in the same rectangle in $M$, and any $v, w \in \mathbb{T}^{2}$. Since $h$ is uniformly continuous, we can take $\delta<\epsilon$ to be small enough such that if $x$ and $y$ are in the same rectangle, and $d(x, y)<\delta$ then $W_{\epsilon}^{u}(y, f) \cap W_{\epsilon}^{s}(x, f)$ and $W_{\epsilon}^{u}(x, f) \cap W_{\epsilon}^{s}(y, f)$ as well as $W_{\epsilon}^{u}(h(y), B) \cap W_{\epsilon}^{s}(h(x), B)$ and $W_{\epsilon}^{u}(h(x), B) \cap W_{\epsilon}^{s}(h(y), B)$ are each precisely one point. We can also choose $\delta$ so that $d(x, y)<\delta$ implies $d(h(x), h(y))<\epsilon$ in $\mathbb{T}^{2}$. Finally, since $h$ is a semiconjugacy we note that $h\left(W_{\delta}^{u}(x, f)\right) \subset W_{\epsilon}^{u}(h(x), B)$ and $h\left(W_{\delta}^{s}(x, f)\right) \subset W_{\epsilon}^{s}(h(x), B)$ for all $x \in M$.

Suppose $h$ is not one-to-one on all rectangles of diameter $\delta$. Then there exist $x$ and $y$ in a rectangle of diameter less than $\delta$ such that $h(x)=h(y)$. But this implies that $W_{\delta}^{u}(y, f) \cap W_{\delta}^{s}(x, f)$ must also map under $h$ to the point $h(x)=h(y)$, since the image must be $W_{\epsilon}^{u}(h(x), B) \cap W_{\epsilon}^{s}(h(y), B)$. Similarly, the point $W_{\delta}^{u}(x, f) \cap W_{\delta}^{s}(y, f)$ must map under $h$ to this same point. Hence all four corners of the picture shown in Figure 1 must map to the same point.

Given a point $z$ in the rectangle, $R$, formed by these four corners, we can consider $W^{u}(z, f, R)$, the unstable line segment through $z$ contained in the rectangle, and $W^{s}(z, f, R)$, the stable line segment through $z$ contained in the rectangle. If $h\left(W^{s}(z, f, R)\right)$ is not on the boundary of $h(R)$, then since $h$ is continuous, there is at least one $z^{\prime} \in W^{u}(z, f, R)$, distinct from $z$, such that $h\left(W^{s}\left(z^{\prime}, f, R\right)\right)=$ $h\left(W^{s}(z, f, R)\right)$ and hence $h(z)=h\left(z^{\prime}\right)$. Indeed, there is a small interval $U$ containing $z$ in $W^{u}(z, f, R)$ with the property that any point in this interval has the same image under $h$ as another point of $W^{u}(z, f, R)$. Because $h$ must send local stable and unstable manifolds for $f$ to local stable and unstable manifolds for $B$, there is a neighborhood of $z \in R$ such that every point has the same image under $h$ as another point on its local unstable manifold.

Since periodic points are dense, there is a periodic point, $p$, in $R$ and another point, $q$, in the local unstable manifold of $p$ such that $h(p)=h(q)$. Consider an iterate of $f, f^{m}$, such that $f^{m}(p)=p$. Henceforth we replace $f$ by $f^{m}$ and 


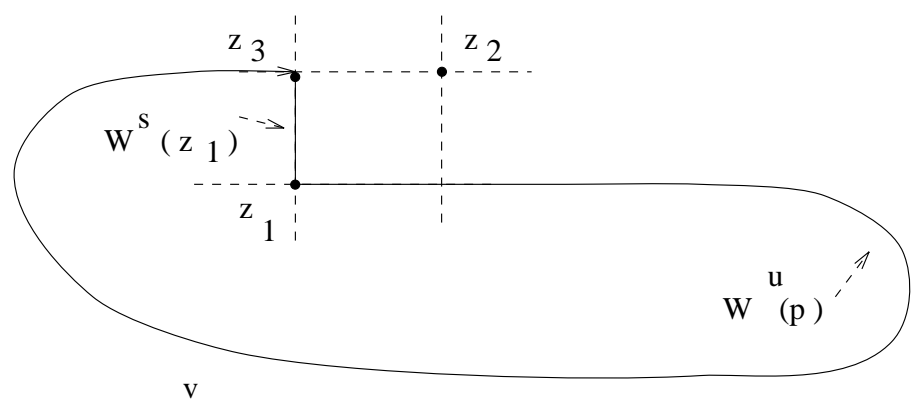

FiguRE 2

$B$ by $B^{m}$. The sequence $f^{k}(q)$ has a convergent subsequence. Let $z$ be a limit point. Then there exist $k_{1}$ and $k_{2}$ such that $d\left(f^{k_{i}}(q), z\right)<\delta / 2$ for $i=1,2$. Hence $d\left(f^{k_{1}}(q), f^{k_{2}}(q)\right)<\delta$. Let $z_{i}=f^{k_{i}}(q) \in W^{u}(p)$ for $i=1,2$. Then $h\left(z_{i}\right)=$ $h\left(f^{k_{i}}(q)\right)=B^{k_{i}}(h(q))=B^{k_{i}}(h(p))=h\left(f^{k_{i}}(p)\right)=h(p)$. Once again, we must have $h\left(W_{\delta}^{s}\left(z_{1}\right) \cap W_{\delta}^{u}\left(z_{2}\right)\right)=h\left(W_{\delta}^{s}\left(z_{2}\right) \cap W_{\delta}^{u}\left(z_{1}\right)\right)=h\left(z_{1}\right)=h\left(z_{2}\right)=h(p)$. Let $z_{3}=W_{\delta}^{s}\left(z_{1}\right) \cap W_{\delta}^{u}\left(z_{2}\right)$.

Consider the picture shown in Figure 2. Consider the loop $v$ that consists of the arc in the unstable manifold of $p$ from $z_{1}$ to $z_{3}$ followed by the small piece of stable manifold from $z_{1}$ to $z_{3}$. This is a closed loop in $M$. Moreover, since $h\left(z_{1}\right)=h\left(z_{3}\right)$, its image under $h$ is the union of two loops, one of which lies completely in the stable manifold of $h(p)$ and the other lies completely in the unstable manifold of $h(p)$. Hence both are contractible and thus $h(v)$ is null-homotopic.

Consider a basis for $H_{1}(M)$ given by eigenvectors and generalized eigenvectors of $f_{* 1}$ such that $e_{1}$ is the eigenvector of $\lambda$. Since $f_{* 1}: H_{1}(M) \rightarrow H_{1}(M)$ and a Markov matrix will have the same eigenvalues up to zeros and roots of unity [8], $\lambda$ is the unique eigenvalue with largest modulus. An element of $H_{1}(M)$ can be expressed as a linear combination of these basis elements. Hence

$$
[v]=a_{1} e_{1}+\sum_{j>1} a_{j} e_{j}
$$

Since $\lambda$ is the unique eigenvalue with largest modulus, we have

$$
\lim _{n \rightarrow \infty} \frac{f_{* 1}^{n}[v]}{\lambda^{n}}=a_{1} e_{1}
$$

The proof is a well known argument. Now

$$
\phi\left(\lim _{n \rightarrow \infty} \frac{f_{* 1}^{n}[v]}{\lambda^{n}}\right)=a_{1} \overline{e_{1}}
$$

where $\overline{e_{1}}=\phi\left(e_{1}\right)$ is the eigenvector for $B$ with eigenvalue $\lambda$. Thus, if we can show that $a_{1} \neq 0$, then we will have obtained a contradiction.

Given two elements $u$ and $v$ of $H_{1}(M, \mathbb{R})$, we can define their inner product, $\langle u, v\rangle$, to be the intersection number of the oriented loops $\alpha$ and $\beta$ where $u=[\alpha]$ and $v=[\beta]$. Let $w$ be a loop that lies mostly in the stable manifold of a periodic point $q^{\prime}$ with angle $2 \pi$ with a small part of the loop in the unstable direction. Do this in such a way that $v$ and $w$ have a non-zero intersection and this intersection is an intersection of the unstable manifold of $p$ with the stable manifold of $q^{\prime}$. Since 
$M$ is oriented, and the stable and unstable foliations are oriented, $v$ and $w$ will always intersect in the same way, hence $\langle[v],[w]\rangle \neq 0$.

Consider a Markov partition for $f$ on $M[2]$. We can construct $v$ such that if $v$ intersects a rectangle, then $v$ completely crosses that rectangle in the unstable direction. The only place where this might not happen is near $z_{1}$, where the loop contains part of the stable manifold of $z_{1}$. This can be avoided by considering where $z_{1}$ lies in the rectangle and taking a $z_{2}$ that lies within the same rectangle. This is possible since $z$ is a limit point. This insures that the part of the loop which is part of the stable manifold lies completely in one rectangle. We can also construct $w$ such that if $w$ intersects a rectangle, then $w$ completely crosses the rectangle in the stable direction. This is possible since $W^{s}\left(q^{\prime}\right)$ is dense in $M$. Let $\mathcal{M}$ be the Markov matrix for our partition.

We have that $f_{* 1}^{n}[v]=\left[f^{n}(v)\right]$. The intersection number of $f^{n}(v)$ and $w$ is the sum

$$
\sum_{i, j} r_{i} s_{j} \mathcal{M}_{i, j}^{n}
$$

where $\mathcal{M}^{n}$ is the Markov matrix for $f^{n}, v$ crosses the $i t h$ rectangle $r_{i}$ times and $w$ crosses the $j t h$ rectangle $s_{j}$ times. By Proposition 1.7, we have

$$
\lim _{n \rightarrow \infty} \frac{\mathcal{M}_{i, j}^{n}}{\lambda^{n}}=c_{i j}
$$

where $c_{i j}$ is a non-zero constant for every $i, j$. This gives us that

$$
\begin{aligned}
\left\langle\lim _{n \rightarrow \infty} \frac{f_{* 1}^{n}[v]}{\lambda^{n}},[w]\right\rangle & =\lim _{n \rightarrow \infty} \frac{\left\langle f_{* 1}^{n}[v],[w]\right\rangle}{\lambda^{n}} \\
& =\lim _{n \rightarrow \infty} \frac{\sum_{i, j} r_{i} s_{j} \mathcal{M}_{i, j}^{n}}{\lambda^{n}} \\
& =\lim _{n \rightarrow \infty} \sum_{i, j} r_{i} s_{j} \frac{\mathcal{M}_{i, j}^{n}}{\lambda^{n}} \\
& =\sum_{i, j} r_{i} s_{j} c_{i, j}>0 .
\end{aligned}
$$

Since the intersection is non-zero, we must have that

$$
\lim _{n \rightarrow \infty} \frac{f_{* 1}^{n}[v]}{\lambda^{n}} \neq 0
$$

Hence $a_{1} \neq 0$ and we have obtained a contradiction to the assumption that $h$ is not one-to-one on a rectangle of diameter less than $\delta$. Since $h$ is continuous and one-toone on these rectangles and the rectangles are compact it follows that $h$ maps such a rectangle homeomorphically onto its image.

Theorem 2.3. If $f: M \rightarrow M$ is a pseudo-Anosov homeomorphism on an orientable surface with oriented unstable manifolds and a quadratic expanding factor, then there is a hyperbolic toral automorphism $B$ on $\mathbb{T}^{2}$ and a map $h: M \rightarrow \mathbb{T}^{2}$ such that $h$ is a semi-conjugacy from $f$ to $B$ and $(M, h)$ is a branched covering space of $\mathbb{T}^{2}$. Moreover the map $h$ restricted to the complement of the singularities of $f$ is a locally affine map from the flat Euclidean structure on $M$ to the natural affine structure on $\mathbb{T}^{2}$. 
Proof. Let $S$ be the set of singularities of $f$ in $M$. Consider $h(S)$ and $h^{-1}(h(S))$. Then by the above, we have that $h: M \backslash h^{-1}(h(S)) \rightarrow \mathbb{T}^{2} \backslash h(S)$ is a covering map. Hence we have shown that $h$ is a branched covering. The fact that $h$ is affine on the complement of $S$ follows from the fact that the stable and unstable foliations of both $f$ and $B$ are uniquely ergodic (see A. Fathi and V. Poénaru, [1]). This is because the Euclidean structure defines a transversally invariant measure for any of these foliations. Unique ergodicity implies that these measures are unique up to a positive scalar multiple. It follows that there is a constant $c$ such that for any segment $I$ of a stable manifold of $f$, the length of $h(I)$ is $c$ times the length of $I$. A similar result holds for segments of unstable manifold (perhaps with a different constant). These facts imply that $h$ is locally affine.

\section{Foliations AND QUADRATIC EXPANSION}

In this section we prove a second characterization of pseudo-Anosov homeomorphisms with quadratic expansion factor in terms of foliations compatible with the flat Euclidean structure associated with the homeomorphism.

Suppose $f: M \rightarrow M$ is a pseudo-Anosov homeomorphism on an orientable surface with oriented stable and unstable manifolds. Given any extended real number $m \in \mathbb{R} \cup\{\infty\}$ we can associate to it a foliation of $M$ with singularities - the same singularities as those of $f$. This is done by specifying the leaf through any non-singular point of $M$ to be the line through that point with slope $m$ in the flat Euclidean structure where the slope is measured with respect to $(x, y)$ axes in the same direction as the oriented unstable and stable manifolds respectively. We choose an orientation of $M$ so that these local coordinates form a positively oriented frame.

Definition 3.1. The slope foliation $\mathcal{F}_{m}$ associated to $m$ and $f$ is the foliation with slope $m$ and singularities at the singularities of $f$ as constructed as above.

Note the foliations $\mathcal{F}_{0}$ and $\mathcal{F}_{\infty}$ are the unstable and stable manifold foliations respectively. In the neighborhood of a singularity the leaves of any $\mathcal{F}_{m}$ look like the leaves of a stable or unstable manifold foliation, i.e. there is some even number of line segments which end in the singularity and all other local leaves are line segments. By convention we will consider the singularity and all the leaf line segments which end at it to be part of the same (global) leaf of the foliation. A leaf containing a singularity like this will be called a singular leaf. Thus every point of $M$ will be on a leaf of $\mathcal{F}_{m}$, but a leaf containing a singularity will not be a manifold as it contains a point, viz. the singularity, which has neighborhood which is not locally Euclidean.

We will be interested in values of $m$, if any exist, for which every leaf of $\mathcal{F}_{m}$ is compact. We will say such a foliation has compact leaves. If $\mathcal{F}_{m}$ has compact leaves it is clear that the complement in $M$ of the (finitely many) leaves containing singularities is a union of disjoint open annuli embedded in $M$. This is because each component of this complement is an open oriented surface foliated by circles and is hence an annulus. Each of these annuli inherits a flat Euclidean structure from $M$, and is isometric to $(0, a) \times S^{1}$ for some interval $(0, a) \subset \mathbb{R}$ and $S^{1}=\{(x, y) \in$ $\left.\mathbb{R}^{2} \mid x^{2}+y^{2}=r^{2}\right\}$ for some $r>0$. We will call $a>0$ the width of the annulus. 
Definition 3.2. We will say that $\mathcal{F}_{m}$ is rational provided it has compact leaves and the ratio of the widths of any two annuli in the complement of the singular leaves is rational.

Theorem 3.3. Suppose $f: M \rightarrow M$ is a pseudo-Anosov homeomorphism on an orientable surface with oriented stable and unstable manifolds, then the following are equivalent:

1) The expansion factor $\lambda$ of $f$ is quadratic.

2) There exists an $m \in \mathbb{R}$ such that the slope foliation $\mathcal{F}_{m}$ of $M$ is rational.

3) There exists a dense set of $m \in \mathbb{R}$ such that the slope foliation $\mathcal{F}_{m}$ of $M$ is rational.

Proof. We first show that 1 ) implies 3). By Theorem 2.3 we can assume that there is a branched covering $h: M \rightarrow \mathbb{T}^{2}$ which is a semiconjugacy from $f$ to a hyperbolic toral automorphism $B$ on $\mathbb{T}^{2}$. Choose any rational slope on $\mathbb{T}^{2}$ and consider the foliation of $\mathbb{T}^{2}$ by circles with this slope. Since $h$ can be chosen to be a local isometry, $h^{-1}$ of this foliation will be an affine foliation with singularities of $M$ which is oriented and transversely oriented. Hence this foliation is $\mathcal{F}_{m}$ for some $m$. Note that this $m$ is not the original rational slope we chose on the torus as that slope was chosen with respect to the standard basis of $\mathbb{R}^{2}$ (where $\mathbb{T}^{2}=\mathbb{R}^{2} / \mathbb{Z}^{2}$ ) and the slope $m$ is measured with respect to axes parallel to the stable and unstable manifolds. Nevertheless the rational slopes on the torus give rise to a dense set of possible choices of $m$ such that $\mathcal{F}_{m}$ has compact leaves.

To show that each such $\mathcal{F}_{m}$ is rational we must show that the annuli in $M$ which form the complement of singular leaves all have widths which are rationally related. But $h$ restricted to one of these annuli is a covering map onto an annulus in $\mathbb{T}^{2}$ which has the same width since $h$ is a local isometry. Thus it suffices to show the widths of the annuli on $\mathbb{T}^{2}$ are rationally related. But these annuli are the components of the complement of the circles in the foliation of $\mathbb{T}^{2}$ which contain the image under $h$ of the singularity set. The image under $h$ of the singularity set is a finite set of periodic (and hence rational) points of the torus. To compare the widths of two of these annuli in the torus we lift them to strips in the universal covering space $\mathbb{R}^{2}$ and compare the widths there. These strips have boundaries with rational slope which pass through at least one point with rational coordinates, namely the lift of a point in the $h$ image of a singularity. Thus the boundaries of these strips all intersect one axis (say the $x$-axis) in rational points. The ratio of the widths of two such strips is the same as the ratio of the lengths of the intervals obtained by intersecting the strips with the $x$-axis. But these intervals have rational endpoints and hence rational lengths. It follows that the foliations $\mathcal{F}_{m}$ are rational as claimed and we have shown that 1) implies 3).

Clearly 3 ) implies 2) so we need only show that 2) implies 1 ). To do this we consider an affine differential form associated to each slope $m$ and defined on $M$ except at singularities.

For each $m$ we define $\omega_{m}$ to be the form given in local coordinates on $M$ by

$$
\omega_{m}=a d x+b d y
$$

where, as before, $x$ and $y$ are Euclidean local coordinates parallel to the (oriented) unstable and stable manifolds respectively, and where $a^{2}+b^{2}=1, \operatorname{sgn}(b)=\operatorname{sgn}(m)$, and $b / a=-1 / m$. We let $\omega_{0}=d y$ and $\omega_{\infty}=-d x$. 
Clearly the form $\omega_{m}$ is well defined on the complement of the singularities of $M$, affine and closed. A straightforward computation shows that if $\Gamma$ is a small closed loop around any singularity in $M$ then $\int_{\Gamma} \omega_{m}=0$. From this it follows that $\omega_{m}$ defines a real cohomology class by the formula

$$
\left\langle\left[\omega_{m}\right],[\gamma]\right\rangle=\int_{\gamma} \omega_{m}
$$

where $[\gamma]$ represents the homology class of a smooth closed loop $\gamma$ in $M$.

Note that

$$
\omega_{m}=a d x+b d y=-a \omega_{\infty}+b \omega_{0}
$$

and that

$$
f^{*}\left(\left[\omega_{\infty}\right]\right)=\lambda\left[\omega_{\infty}\right] \quad \text { and } \quad f^{*}\left(\left[\omega_{0}\right]\right)=\lambda^{-1}\left[\omega_{0}\right] .
$$

It follows that we need only show the existence of $m$ and $\beta$ such that $\beta\left[\omega_{m}\right]$ is a rational cohomology class, i.e. that $\left\langle\beta\left[\omega_{m}\right], u\right\rangle$ is rational for every integer homology class $u$. This is sufficient to prove our theorem because then it will follow that $\beta\left[\omega_{m}\right]$ and $f^{*}\left(\beta\left[\omega_{m}\right]\right)$ form a rational basis of a two-dimensional rational vector space which is invariant under $f^{*}$. Some integer multiples of these two cohomology classes will be integer cohomology classes, and if these multiples are used as a basis of this two-dimensional rational vector space then the matrix of $f^{*}$ with respect to this basis will be integral. Since $\lambda$ is an eigenvalue of this two-by-two integer matrix we can conclude that $\lambda$ is the root of a monic quadratic polynomial with integer coefficients, and hence quadratic.

We now complete the proof by showing that whenever $m \in \mathbb{R}$ is such that $\mathcal{F}_{m}$ is a rational foliation then there exists a $\beta$ such that $\beta\left[\omega_{m}\right]$ is a rational cohomology class. The widths of the annuli making up the complement of the singular leaves of $\mathcal{F}_{m}$ are rationally related so there exists a $\beta$ such that $\beta$ times the width of any of these annuli is rational.

Now if $\alpha$ is an arc lying in one of these annuli and crossing it once (i.e. going from one boundary to another) then $\int_{\alpha} \beta \omega_{m}$ equals $\pm \beta$ times the width of the annulus, i.e., a rational number. Also if $\alpha$ is an arc lying in one of these annuli and starting and ending in the same boundary component then $\int_{\alpha} \beta \omega_{m}=0$. Since any smooth closed loop $\gamma$ in $M$ is homotopic to one which can be decomposed into arcs of these

two types, it follows that $\int_{\gamma} \beta \omega_{m}$ is rational and hence that the cohomology class $\beta\left[\omega_{m}\right]$ is rational.

\section{REFERENCES}

[1] Fathi, A. and Poénaru, V., Exposé 12: Theoremes d'unicit'e des diffeómorphismes pseudoAnosov, Travaux de Thurston sur les Surfaces, Astérisque 66-7 Société Mathématique de France, Paris (1979).

[2] Fathi, A. and Shub, M., Exposé 10: Some Dynamics of Pseudo-Anosov Diffeomorphisms, Travaux de Thurston sur les Surfaces, Astérisque 66-7 Société Mathématique de France, Paris (1979).

[3] Franks, John, Global Analysis, Proceedings of Symposia in Pure Mathematics 14, American Mathematical Society, Providence, Rhode Island (1970).

[4] Franks, John, Invariant Sets of Hyperbolic Toral Automorphisms, American Journal of Mathematics 99, No. 5, 1089-1095, John Hopkins University Press (1977). MR 58:2891

[5] Gantmacher, F.R., The Theory of Matrices, Volume Two, Chelsea Publishing Company, New York (1959). MR 21:6372c 
[6] Karlin, Samuel and Taylor, Howard, A First Course in Stochastic Processes, second edition, Academic Press, New York (1975). MR 50:8668

[7] Masur and Smillie, Hausdorff Dimension of Sets of Nonergodic Measured Foliation, Annals of Mathematics, 2nd Series 134 (1991) no. 3, pp. 455-543. MR 92j:58081

[8] Rykken, E., Expanding Factors for Pseudo-Anosov Homeomorphisms (preprint) (1997).

Department of Mathematics, Northwestern University, Evanston, Illinois 60208

Current address, J. Franks: Department of Mathematics, Northwestern University, Evanston, Illinois 60208

E-mail address: john@math.nwu.edu

Current address, E. Rykken: Department of Mathematics, Indiana University Northwest, Gary, Indiana 46408

E-mail address: erykken@iunhaw1.iun.indiana.edu 